\title{
Amerikanische Literatur zum Supreme Court - Lücken bei der Forschung zum Bundesverfassungsgericht
}

Dass Verfassungsgerichtsbarkeit integraler Bestandteil des politischen Teils von „government" ist (Dahl 1958) und damit auch der permanenten politischen Analyse bedarf, muss in den USA in keiner langatmigen Erörterung über das dialektische Verhältnis von Recht und Politik erst noch begründet werden. Lässt man den Blick über die amerikanische Literatur schweifen, dann kann man das schon bei einem flüchtigen Besuch in einer guten Buchhandlung einer mittelgroßen Provinzstadt neidvoll feststellen. Denn hier gibt es Bücher zum Supreme Court - zumeist einsortiert unter der Rubrik ,,politics/government“" -, die bei uns noch nicht einmal in hoch spezialisierten Universitätsbuchhandlungen stehen. Das spiegelt sich auch in der aktuellen Literaturlage wider, die wegen der politischen Konflikte um den zukünftigen Kurs der Rechtsprechung anlässlich der Besetzung vakanter Richterstellen in den letzten Jahren noch einmal einen regelrechten Publikationsschub erhalten hat. Dabei lassen sich drei verschiedene „Literaturgattungen“ unterscheiden, die - für hiesige Verhältnisse kaum vorstellbar - nicht nur immer wieder mit „Bestseller-Auflagen“ verbunden sind, sondern für die sich in der Literatur zum Bundesverfassungsgericht auch zum Teil oder sogar überhaupt keine Entsprechungen finden lassen.

So gibt es erstens zahlreiche Biografien und Übersichtsdarstellungen, in denen der rechtspolitische Einfluss einzelner Richterpersönlichkeiten in zentralen, Weichen stellenden Epochen, Entscheidungsreihen und Rechtsprechungsphasen zeitgeschichtlich eingeordnet ${ }^{1}$ bzw. in historischen Querschnittsanalysen (Irons 1999; Rosen 2007) herausgearbeitet wird: Man denke für die Zeit nach 1945 etwa an die berühmten Fälle wie „Brown vs. Board of Education“ zur Rassentrennung in der Ära des „Warren-Court“ der 50er-Jahre, an die bis heute wirkmächtige und umkämpfte, seinerzeit vom liberal gewandelten Richter Blackmun formulierte Entscheidung „Roe vs. Wade“ von 1973 zur Abtreibungsfrage - aber auch an den Bürgerrechtler Thurgood Marshall, 1967 als erster afro-amerikanischer Richter berufen, und Sandra Day O'Connor, erste Richterin am Supreme Court ${ }^{2}$, der bald nach ihrer Berufung 1981 über Jahre hinweg als sog. „swing vote“ eine zentrale Machtposition zufiel. Dies geschieht überaus quellengesättigt und unter Auswertung von Archiven/Nachlässen, z. T. auch in eher populistisch verfassten Polemiken (z. B. Levin 2002), und wegen des hohen Aufmerksamkeitswerts regelmäßig auch in journalistischen Analysen der Tagespresse und politischen Magazinen (z. B. Talbot 2005; Cohen 2006).

In Deutschland ist dieser Literaturtypus dagegen fast gar nicht präsent: Von wenigen Ausnahmen abgesehen (wie etwa anlässlich des aktuellen Streits um die Nominierung von

1 Z. B. Powe 2000; Schwartz 2002; Greenhouse 2005; Newton 2006.

2 Allein über Marshall und O'Connor sind in den letzten Jahren jeweils mehr als ein Dutzend Bücher erschienen. 
Horst Dreier) erfährt man über Richter am Bundesverfassungsgericht in der Qualitätspresse - wenn überhaupt - allenfalls am Rande (z. B. Müller 2006). Porträts einzelner Persönlichkeiten in fachwissenschaftlichen Zeitschriften/Jahrbüchern erscheinen meist nur im Falle wohlmeinender Nachrufe (z. B. Lerche 2007). Ausführliche Richterbiografien, in denen im oben genannten Sinne eine Verbindung von Persönlichkeit, staatstheoretischem Vorverständnis und Richterpraxis in zeitgeschichtlicher Perspektive gewagt wird, ${ }^{3}$ gibt es fast genauso wenig wie die historische Einordnung zentraler Entscheidungen des Bundesverfassungsgerichts. ${ }^{4}$

Die juristische Literatur bleibt überwiegend an dogmatischen Fragestellungen interessiert, ${ }^{5}$ in denen sozusagen per definitionem der Faktor „Persönlichkeit“ ebenso wenig eine Rolle spielen kann und darf wie der Faktor „Politik“. Und in der Politikwissenschaft wird das Bundesverfassungsgericht selbst in den Standardeinführungen zum Regierungssystem seit Jahren eher randständig abgehandelt ${ }^{6}$ - von vergleichenden Analysen zu ausländischen Verfassungsgerichten ganz zu schweigen (vgl. aber z. B. Lhotta 1995; aktuell Höreth 2008) -, obwohl es gerade im deutschen politischen System besonders mächtig, ja vielleicht noch mächtiger sogar als der US Supreme Court ist: das Bundesverfassungsgericht sozusagen als „blinder Fleck“ politikwissenschaftlicher Forschung. ${ }^{7}$ Es kann daher nicht verwundern, dass in der zweibändigen, voluminösen Festschrift zu dessen 50-jährigem Bestehen gerade mal ein Politologe vertreten ist (nämlich von Beyme 2001) - für die amerikanische Forschungslandschaft nahezu undenkbar. Von Beyme beklagt diese Eigentümlichkeit dann auch zu Recht.

Die Dominanz herrschender systemtheoretischer Zugänge und Policy-Analysen hat in den stark sozialwissenschaftlich ausgerichteten Arbeiten so nicht nur eine Abkoppelung von normativen (und damit auch verfassungsrechtlichen) Fragen verursacht, sondern zudem eine Wahrnehmung des Faktors „Persönlichkeit“ - hier ohnehin als konservativ-altmodischer Ansatz verschrien - völlig versperrt. Der mehrheitlich ,ahistorisch“ arbeitende „Mainstream" hat sich daher von der Zeitgeschichte ebenso verabschiedet wie vom öffentlichen Recht. Politologen, die zeitgeschichtlich arbeiten, sind zumeist ganz in die Geschichtswissenschaft abgewandert bzw. werden nur als ,fachfremde“" Historiker wahrgenommen. ${ }^{8}$ So muss der eigentlich ganz selbstverständliche politische Faktor „Persönlichkeit“" in der deutschen Politikwissenschaft überhaupt erst mühsam „,neu“ entdeckt werden (Rosenberger 2005; Hartmann 2007).

3 Vgl. aber z. B. Wiegandt 1995; mit Einschränkung Großfeld/Roth 1995; aktuell und kurz auch Baldus 2007.

4 Vgl. aber z. B. Henne/Riedlinger 2005; z. T. auch Grigoleit 2004; mit Einschränkung Wesel 2004.

5 Es bleibt zu hoffen, dass das von Thomas Vormbaum an der Fernuniversität Hagen initiierte Projekt der Juristischen Zeitgeschichte diese Lücke wenigstens z. T. schließen könnte, wenngleich es im Schwerpunkt nicht auf die Verfassungsrechtsprechung nach 1945 zielt, sondern auf die allgemeine Rechtsgeschichte seit dem 19. Jahrhundert; gleichwohl wird im gleichnamigen Jahrbuch und in dem neu gegründeten „Journal“ auch dies thematisch miterfasst.

6 So von Beyme 2001; vgl. Seibel 2003; Ausnahme hiervon Pilz/Ortwein 2008. Hier werden auch einzelne zentrale Entscheidungen des BVerfG miteinbezogen.

7 Inwieweit ein Trendwechsel zu beobachten ist, bleibt abzuwarten; zur Literaturlage vgl. m. w. N.: van Ooyen/Möllers 2006; Vorländer 2006; van Ooyen 2009 (i. E.).

8 So z. B. im Falle von Arnulf Baring und Hans-Peter Schwarz. 
Als Solitär und eigener Typus in der amerikanischen Literatur zum Supreme Court ragt der „Oxford Guide“ heraus (Hall/Ely/Grossmann 2005), mit seinen über 1200 eng bedruckten, großformatigen Seiten. Hinsichtlich Umfang, enzyklopädischer Vollständigkeit und Interdisziplinarität der Konzeption gibt es auf dem deutschen Markt auch hier nichts Vergleichbares. Es liegen zwar z. Zt. drei handbuchartig konzipierte Bücher zum Bundesverfassungsgericht vor, die dies jeweils aber nicht erfüllen können bzw. wollen. ${ }^{9}$ Schon die durch die beruflichen Qualifikationen der Herausgeber vertretenen Fächer sind für deutsche Verhältnisse überraschend, da gerade nicht durch das Staats- und Verfassungsrecht dominiert: Der federführende Herausgeber Hall lehrt Geschichte, Ely Recht und Geschichte, Grossmann Politikwissenschaft. In ihnen spiegelt sich wider, was auch für die rund 350 beteiligten Autoren und Autorinnen gilt: drei Fächer, die nahezu gleichberechtigt vertreten sind. Als Wörterbuch konzipiert erlaubt der „Oxford Guide“ einen schnellen, lexikalischen Informationszugriff auf nahezu alle Dinge, die bei der über 200-jährigen Geschichte des Supreme Court von Interesse sind: Es finden sich - oft in der Ausführlichkeit eines Handbuchartikels - Richterporträts genauso wie Besprechung und Einordnung der „leading cases“, verfassungstheoretische Grundfragen, Analysen politischer Einflussfaktoren und historischer Traditionsstränge. ${ }^{10}$ Die zweite Auflage bringt dies insgesamt auf den aktuellen Stand, sodass sich gegenüber der Erstauflage auch die Rechtsprechung von 1992 bis 2004 nachschlagen lässt - darunter z. B. die mit der Präsidentenwahl verbundene und auch im Gericht selbst höchst umstrittene Entscheidung „Bush v. Gore“ zur Stimmenauszählung.

Schließlich finden sich in der amerikanischen Literatur „Inside-Politics-Analysen“, die die Arbeitsweisen und -abläufe, vor allem aber auch die internen politischen Machtkämpfe um Richtungsentscheidungen sowie persönliche „Befindlichkeiten“ offenlegen - entweder durch journalistische Recherchen oder durch Berichte ehemaliger "law clerks" des Supreme Courts, die als persönliche Assistenten der jeweiligen Richter etwa den Wissenschaftlichen Mitarbeitern des Bundesverfassungsgerichts entsprechen (vgl. z. B. den ausführlichen Insider-Bericht des früheren Blackmun-clerk Lazarus 2005). Im Unterschied zu den USA wissen wir jedoch „nicht viel über die Tätigkeit der WiMis. Sie bewegen sich im Arkanum des Kammer-Gerichts“ und „,[a]mtierende Bundesverfassungsrichter haben wenig Interesse, die wahre Rolle der WiMis darzustellen, schmälerten sie doch dadurch ihr eigenes Machtverständnis“" (so der im Bereich des Verfassungsprozessrechts erfahrene Zuck 2006: 287). Hält man bei aller Unterschiedlichkeit die internen Abläufe bei Supreme Court und Bundesverfassungsgericht wenigstens grundsätzlich für vergleichbar - und führt man sich die permanente Überlastung des Bundesverfassungsgerichts gerade im Bereich der Verfassungsbeschwerden vor Augen (z. B. im Jahr 2004: 5434; Gusy 2006: 203) -, so lässt sich angesichts der Lektüre dieser „Clerk-Berichte“ erahnen, welche Steuerungsmacht dem sog. „Dritten Senat“ zufällt. Denn es ist ,undenkbar, dass er [der Richter, RvO] die Fülle der häufig umfangreichen, ungegliederten [...] Verfassungsbeschwerden gelesen hat (und alle dazugehörigen Gerichtsentscheidungen und sonstigen Unterlagen)“. „Was er kennt, ist das Votum des

9 Badura/Dreier 2001; van Ooyen/Möllers 2006; Menzel 2000.

10 Nebst über hundert Seiten Anhang, darunter insb. ein umfangreiches Stichwortverzeichnis sowie ein vollständiges Verzeichnis aller Richternominierungen und -ernennungen. 
WiMis“ - in vielen Fällen gilt daher: „Die richterliche Verantwortung muss blanko übernommen werden“" (Zuck 2006: 288).

In ähnlicher Weise bleiben auch die internen Abläufe der Richterwahl beim Bundesverfassungsgericht im Dunkeln. Dagegen existiert in den USA eine breitere journalistische Berichterstattung, die sich nicht nur in der Tagespresse regelmäßig mit den Vorgängen rund um den Supreme Court beschäftigt, sondern auch ausführliche, sorgfältig recherchierte Einblicke, z. T. sogar mit Bestsellerauflage, in die machtpolitischen Prozesse gewährt (vgl. schon Woodward/Armstrong 2005; aktuell Toobin 2007). Exemplarisch ist hier eine Arbeit der Journalistin Greenburg zu nennen (2008), ${ }^{11}$ in der mit der Hilfe von mehr als hundert Interviews mit Richtern, „,clerks“, Senatoren sowie Mitarbeitern des Weißen Hauses und des Justizministeriums die politischen Prozesse und Strategien im Umfeld des Weißen Hauses seit den 80er-Jahren rekonstruiert werden. Besondere Bedeutung kommt dabei den Richternominierungen unter Reagan, Bush I und Bush II zu, da die Möglichkeit politischer Einflussnahme seitens der konservativ-religiösen Rechten innerhalb der Republikaner groß schien, die gesamte Richtung der noch infolge der Warren- und Burger-Ära „spät-liberalen“ Rechtsprechung des Supreme Courts endlich zu kippen.

Greenburgs Buch mag als primär journalistisch angelegte Reportage nicht immer strengen Standards wissenschaftlicher Zurückhaltung genügen, ${ }^{12}$ verdeutlicht aber gerade durch diesen Zugang etwas ganz Banales, das in „hochwissenschaftlichen“ Analysen schnell völlig unterzugehen droht: Im und um den Supreme Court geht es einfach zu wie ,im richtigen Leben“. Dabei wird vor allem zweierlei deutlich: eine nicht völlig berechenbare, da auch durch die jeweilige Persönlichkeit stark beeinflusste Eigendynamik innerhalb des Gerichts, die z. B. die ursprünglich eher konservative, auf der Linie von Rehnquist liegende Richterin O'Connor in Reaktion auf konservative Dominanzversuche im Laufe der Zeit nach „links“ wandern ließ, bis ihr als ,swing vote“ zwischen liberalem und konservativem Richterlager die Schlüsselposition bei Patt-Situationen zufiel. Zweitens zeigt sich, eher mit Blick auf die strategischen Kalküle innerhalb des Weißen Hauses, wie komplex und situativ die Nominierungsverfahren sind, sodass sich auch hier kein generelles Muster vorhersehen lässt. Eine erfolgreiche Nominierung ist nicht einfach bloß abhängig von der fachlichen Qualifikation, ${ }^{13}$ präsidialen Vorgaben ${ }^{14}$ und den jeweiligen Mehrheitsverhältnissen im Senat. Von Fall zu Fall schwer wägbare Faktoren sind auch: die persönlichen Netzwerke der präsidialen Berater, die die Listen potenzieller Kandidaten zusammenstellen, Erfahrungen aus gescheiterten

11 Greenburg war früher für die Chicago Tribune tätig und ist jetzt bei ABC News.

12 Demgegenüber reichen vergleichbare deutsche Arbeiten längst nicht an das kritisch-investigative Niveau heran; vgl. z. B. Deckenbach 2003.

13 In der Regel: Abschluss an einer Elite-Universität, Berufserfahrung, insb. auch als Richter an Bundesgerichten; ausgewiesene verfassungsrechtliche Kenntnisse, da die „hearings“ mittlerweile regelrechten Prüfungscharakter haben.

14 Z. B. die ursprüngliche Vorgabe von Bush II, für die Nachfolge von O’Connor eine Frau oder einen Angehörigen einer (spanischen) Minderheit zu nominieren, sodass Harriet Miers ins Rennen geschickt wurde, die dann aber prompt „durchfiel“". 
Nominierungen und das „Timing“, ${ }^{15}$ die Einschätzung des möglichen öffentlichen Widerstands, mit Blick auf die Öffentlichkeit des Verfahrens auch mögliche „schwarze Flecken“ auf der Weste der Kandidaten, ${ }^{16}$ die jeweilige Stärke der intern rivalisierenden Parteiflügel, zu berücksichtigende „Quoten“ politischer Repräsentation, ${ }^{17}$ die interne Machtbalance des Supreme Courts zwischen „Liberalen“ und „Konservativen“, ${ }^{18}$ die staatstheoretischen und verfassungsrechtlichen Grundpositionen der Kandidaten sowie ihr persönliches Agieren beim ,hearing“, ${ }^{19}$ insb. bei heiklen Fragen wie „Abtreibung“ usw. - schließlich auch im Hinblick auf die lebenslange Einflussmöglichkeit infolge der zeitlich nur durch Tod oder Rücktritt beschränkten Amtsdauer: Alter und Gesundheit ${ }^{20}$.

Von einem Offenlegen solcher Mechanismen kann in Deutschland aber keine Rede sein. Zwar wird in der Regel einer breiteren politisch interessierten Öffentlichkeit bei den Verfassungsrichterwahlen noch bekannt, welche Richter welcher parteipolitischen Grundorientierung zuzuordnen ist. Staatstheoretische und damit politische Vorverständnisse zu zentralen, die Bürger direkt betreffenden Fragen des Verfassungsrechts - man führe sich z. B. die aktuellen Herausforderungen im Bereich der Inneren Sicherheit vor Augen - bleiben aber schon im Dunkeln. Das, obwohl so manche wichtige Entscheidung des Bundesverfassungsgerichts nur mit denkbar knappem Mehrheitsbeschluss ausfällt. So ist man generell „von der Möglichkeit, die Öffentlichkeit in den Prozess der Richterwahlen einzubeziehen, weit entfernt. ,Verschwiegenheit' heißt die Devise“" (Landfried 2006: 241).

Das liegt nicht allein an der Ausgestaltung des Wahlverfahrens in Bundestag und Bundesrat, bei dem sich die Kandidaten eben nicht einem - zwar immer wieder geforderten - ,hearing“ unterziehen müssen (vgl. schon Häberle 1994; aktuell Montag 2008). Im umgekehrten Falle ist es auch nicht allein dem Umstand geschuldet, dass die Nominierung in den USA eine zunächst reine präsidiale Kompetenz ist und es dort zudem keine zeitliche „Deckelung“ des Amts gibt, da ja auf Lebenszeit ernannt wird. So mag sich zwar immer mal die historisch besondere und höchst politisierte Situation ergeben, dass ein Präsident infolge der kurzfristigen Vakanz gleich mehrerer Stellen tatsächlich die Verfassungspolitik des Supreme Courts über Jahrzehnte hinaus beeinflussen kann. In den USA aber scheint man sich trotz einer sogar stärker verankerten Tradition der „Gewaltentrennung“ viel bewusster darüber zu sein, wie sehr Verfassungsgerichtsbarkeit und Politik zusammenhängen, wie sehr also mit der Zusammensetzung des Gerichts (rechts-)politische Implikationen verbunden sind, die auch unmittelbar auf die persönliche Lebensgestaltung und individuelle Freiheit durchschlagen - und das

15 So unter Bush I die Nominierung von David Souter, der sich zum großen Ärgernis der Konservativen als „liberal“ entpuppte; auch die vor allem an der internen Rechten gescheiterte Nominierung von Harriet Miers während der durch „Irak-Krieg“ und „Hurricane Katrina“ geschwächten Führungsphase von Bush II.

16 Z. B. mutmaßlicher früherer Haschischkonsum des Kandidaten Douglas Ginsburg.

17 So wohl nicht nur ein Faktor im Falle des Afro-Amerikaners Thomas, sondern auch bei Antonio Scalia als Repräsentant der wichtigen Italo-amerikanischen Minderheit; ebenso bei der Nominierung von O'Connor durch Reagan.

18 Im strategischen Kalkül kann das sogar dazu führen, dass ein Präsident keinen völlig auf seiner „Linie“ liegenden Kandidaten vorschlägt, um nicht ein „swing-vote“ durch die sich daraus ergebende interne Machtverschiebung vollständig in das andere Lager zu ,treiben“.

19 So beim ,hearing“ der gescheiterten Nominierung von Robert Bork.

20 Z. T. über 30 Jahre, z. B. der konservative Chief Justice Rehnquist. 
nicht nur im Falle der höchst kontroversen Fragen von Abtreibung, Todesstrafe und Minderheiten.

So wird um die einzelnen Richter fast immer öffentlich politisch gekämpft: im Senat, der letztendlich zustimmen muss, seitens der in ihren Rechten/Positionen sich bedroht fühlenden zivilgesellschaftlichen Gruppen von links und rechts, in der Presse, in den Flügeln der Parteien, ja sogar in der wissenschaftlichen Auseinandersetzung. Insofern ist die Rechtskultur in den USA viel politischer und konfliktorientierter - und daher auch demokratischer - während in Deutschland infolge antidemokratischer Kontinuitätslinien der Mythos vom „unpolitischen“ Recht, das jenseits gesellschaftlicher Interessen stehe, wirkmächtig geblieben ist (Sontheimer/ Bleek 1999: 184 ff.). ${ }^{21}$ Diese „Entpolitisierung“ des Staats- und Verfassungsrechts durch scheinneutrale und -objektive juristische Argumentationen und Formalismen kann nicht anders als „obrigkeitsstaatlich“ genannt werden (vgl. schon Radbruch 1930: 289, bzgl. der vermeintlichen Überparteilichkeit des Staatsoberhaupts). Aus wissenschaftlicher Sicht bleibt daher enormer Nachholbedarf, um endlich zu einem tieferen und schließlich dann auch breiter verankerten politischen Verständnis der Verfassungsgerichtsbarkeit durchzudringen. Das gilt erst recht angesichts der Tatsache, dass dieser Mythos bisweilen gerade vom Bundesverfassungsgericht selbst gepflegt wird - sei es, um sich hierüber eine zusätzliche Legitimationsreserve und Machtressource zu erschließen, oder sei es, dass man hier, „gefangen“ in den Traditionen deutscher Staatslehre, dem Mythos sogar selbst aufsitzt (vgl. m. w. N. van Ooyen 2005, 2008).

\section{Literatur}

Badura, Peter/Dreier, Horst (Hrsg.), 2001: Festschrift 50 Jahre Bundesverfassungsgericht, Bd. 1, Tübingen.

Baldus, Manfred, 2007: Wer war und wofür steht Martin Drath?; in: Recht und Politik 43 (2), 86-96.

von Beyme, Klaus, 2001: Das Bundesverfassungsgericht aus der Sicht der Politik- und Gesellschaftswissenschaften; in: Badura/Dreier 2001, 493-505.

Cohen, Adam, 2006: What Chief Justice Roberts Forgot in His First Term: Modesty, in: New York Times, 09.07.06.

Dahl, Robert A., 1958: Decision-Making in a Democracy: The Supreme Court as a National Policy-Maker: in: Journal of Public Law 6 (2), 279-295.

Deckenbach, Karin, 2003: Jutta Limbach. Eine Biografie, Düsseldorf.

Greenburg, Jan Crawford, 2008: Supreme Conflict. The Inside Story of the Struggle for Control of the United States Supreme Court, ed. with a new afterword, New York.

Greenhouse, Linda, 2005: Becoming Justice Blackmun. Harry Blackmun's Supreme Court Journey, New York.

21 Mit Blick auf die politischen Implikationen von Recht und Verfassungsgerichtsbarkeit vgl. aber auch schon die ideologiekritischen Arbeiten von Hans Kelsen; m. w. N.: van Ooyen 2003. 
Grigoleit, Klaus J., 2004: Bundesverfassungsgericht und deutsche Frage. Eine dogmatische und historische Untersuchung zum judikativen Anteil der Staatsleitung, Tübingen.

Großfeld, Bernhard/Roth, Herbert (Hrsg.), 1995: Verfassungsrichter. Rechtsfindung am U.S. Supreme Court und am Bundesverfassungsgericht, Münster/Hamburg.

Gusy, Christoph, 2006: Die Verfassungsbeschwerde; in: van Ooyen/Möllers 2006, 201-214. Häberle, Peter, 1994: Bundesverfassungsrichter-Kandidaten auf dem Prüfstand? Ein Ja zum Erfordernis „öffentlicher Anhörung“; in: Bernd Guggenberger/Andreas Meier (Hrsg.), Der Souverän auf der Nebenbühne, Opladen, 131-133.

Hall, Kermit L./Ely, James W., Jr. /Grossmann, Joel B. (Hrsg.), 2005: The Oxford Guide to the Supreme Court of the United States, New York (2. Aufl.).

Hartmann, Jürgen, 2007: Persönlichkeit und Politik, Wiesbaden.

Henne, Thomas/Riedlinger, Arne (Hrsg.), 2005: Das Lüth-Urteil aus (rechts-)historischer Sicht. Die Konflikte um Veit Harlan und die Grundrechtsjudikatur des Bundesverfassungsgerichts, Berlin.

Höreth, Marcus, 2008: Die Selbstautorisierung des Agenten: der Europäische Gerichtshof im Vergleich zum U.S. Supreme Court, Baden-Baden.

Irons, Peter, 1999: A People's History of the Supreme Court, New York.

Landfried, Christine, 2006: Die Wahl der Bundesverfassungsrichter und ihre Folgen für die Legitimität der Verfassungsgerichtsbarkeit; in: van Ooyen/Möllers, 229-241.

Lazarus, Edward, 2005: Closed Chambers. The Rise, Fall, and Future of the Modern Supreme Court, New York (Neuauflage; ursprl. 1998).

Lerche, Peter, 2007: Europäische Staatsrechtslehrer. Der Wissenschaftler Konrad Hesse; in: Jahrbuch des öffentlichen Rechts der Gegenwart 55, 455-461.

Levin, Mark R., 2002: Men in Black. How the Supreme Court is Destroying America, New York.

Lhotta, Roland, 1995: Imperiale Verfassungsgerichtsbarkeit und Föderalismus, Baden-Baden.

Menzel, Jörg, 2000: Verfassungsrechtsprechung. Hundert Entscheidungen des Bundesverfassungsgerichts in Retrospektive, Tübingen.

Montag, Jerzy, 2008: Transparenz und Legitimität. Notwendige Reform der Wahl der Richterinnen und Richter zum Bundesverfassungsgericht; in: Recht und Politik 44 (3), 139-145.

Müller, Reinhard, 2006: Schonende Besetzung. Der neue Verfassungsrichter Eichberger; in: FAZ, 08.04.06.

Newton, Jim, 2006: Justice for All. Earl Warren and the Nation He Made, New York.

van Ooyen, Robert Chr., 2003: Der Staat der Moderne, Berlin.

van Ooyen, Robert Chr., 2005: Der Begriff des Politischen des Bundesverfassungsgerichts, Berlin.

van Ooyen, Robert Chr., 2008: Die Staatstheorie des Bundesverfassungsgerichts und Europa, Baden-Baden (2. Aufl.).

van Ooyen, Robert Chr., 2009: Die Unhintergehbarkeit des Politischen in der Verfassungsgerichtsbarkeit; in: Zeitschrift für Politik (i. E.).

van Ooyen, Robert Chr./Möllers, Martin H. W. (Hrsg.), 2006: Das Bundesverfassungsgericht im politischen System, Wiesbaden. 
Pilz, Frank/Ortwein, Heike, 2008: Das politische System Deutschlands, München (4. Aufl.). Powe, Lucas A., Jr., 2000: The Warren Court and American Politics, Cambridge.

Radbruch, Gustav, 1930: Die politischen Parteien im System des deutschen Verfassungsrechts; in: Gerhard Anschütz/Richard Thoma (Hrsg.), Handbuch des Deutschen Staatsrechts, Bd. 1, Tübingen, 285-294.

Rosen, Jeffrey, 2007: The Supreme Court. The Personalities und Rivalries that Defined America, New York.

Rosenberger, Sigrid E., 2005: Der Faktor Persönlichkeit in der Politik, Wiesbaden.

Schwartz, Herman (Hrsg.), 2002: The Rehnquist Court. Judicial Activism on the Right, New York.

Seibel, Wolfgang, 2003: Suchen wir immer an der richtigen Stelle?; in: PVS 44, 217-228.

Sontheimer, Kurt/Bleek, Wilhelm, 1999: Grundzüge des politischen Systems der Bundesrepublik Deutschland, München (11. Aufl.).

Talbot, Margaret, 2005: The Scalia Court; in: The New Yorker, 28.03.05.

Toobin, Jeffrey, 2007: The Nine. Inside the Secret World of the Supreme Court, New York.

Vorländer, Hans (Hrsg.), 2006: Die Deutungsmacht der Verfassungsgerichtsbarkeit, Wiesbaden.

Wesel, Uwe, 2004: Der Gang nach Karlsruhe. Das Bundesverfassungsgericht in der Geschichte der Bundesrepublik, München.

Wiegandt, Manfred H., 1995: Norm und Wirklichkeit. Gerhard Leibholz (1901-1982) - Leben, Werk und Richteramt, Baden-Baden.

Woodward, Bob/Armstrong, Scott, 2005: The Brethren. Inside the Supreme Court, New York (Neuauflage, ursprgl. 1979).

Zuck, Rüdiger, 2006: Die Wissenschaftlichen Mitarbeiter des Bundesverfassungsgerichts, in: van Ooyen/Möllers 2006, 283-292.

Korrespondenzanschrift:

ORR Dr. Robert Chr. van Ooyen

Fachhochschule des Bundes für öffentliche Verwaltung

FB BPol - Gesellschaftswissenschaften

Ratzeburger Landstraße 4

23562 Lübeck

E-Mail: robert.vanooyen@polizei.bund.de

Der Autor ist Dozent an der Fachhochschule des Bundes. 